\title{
Correction
}

\section{Correction: Emrich et al., Distributed Patterns of Activity in Sensory Cortex Reflect the Precision of Multiple Items Maintained in Visual Short-Term Memory}

In the article "Distributed Patterns of Activity in Sensory Cortex Reflect the Precision of Multiple Items Maintained in Visual Short-Term Memory” by Stephen M. Emrich, Adam C. Riggall, Joshua J. LaRocque, and Bradley R. Postle, which appeared on pages 6516-6523 of the April 10, 2013 issue, the following paragraph was omitted from the Materials and Methods section:

\section{Data acquisition and preprocessing}

Whole brain images were acquired with the 3T scanner (Discovery MR750; GE Healthcare) at the Lane Neuroimaging Laboratory at the University of Wisconsin-Madison. High-resolution T1-weighted images were acquired for all subjects with an FSPGR sequence (8.132 ms TR, $3.18 \mathrm{~ms}$ TE, $12^{\circ}$ flip angle, 156 axial slices, $256 \times 256$ in-plane, $1.0 \mathrm{~mm}$ isotropic). BOLD-sensitive data were acquired using a gradient-echo, echoplanar sequence ( $2 \mathrm{~s}$ TR, $25 \mathrm{~ms}$ TE) within a $64 \times 64$ matrix (39 sagittal slices, $3.5 \mathrm{~mm}$ isotropic). Six runs, each lasting $13 \mathrm{~min}$ (390 volumes), were obtained for each subject. The first three TRs from each run were removed before analysis to achieve a steady state of tissue magnetization.

DOI: $10.1523 / J N E U R O S C I .1772-13.2013$ 